
\title{
3 Research Square \\ Diffraction of Waves by a Planar Junction of Impedance and Perfect Electromagnetic Conductor Half-Planes
}

Husnu Deniz Basdemir ( $\nabla$ denizbasdemir@gmail.com )

Konya Food and Agriculture University https://orcid.org/0000-0002-2797-3772

\section{Research Article}

Keywords: Diffraction theory, Impedance surface, Perfect electromagnetic conductor, Scattering theory.

Posted Date: March 12th, 2021

DOl: https://doi.org/10.21203/rs.3.rs-283718/v1

License: (c) (i) This work is licensed under a Creative Commons Attribution 4.0 International License.

Read Full License

Version of Record: A version of this preprint was published at Optical and Quantum Electronics on August 17th, 2021. See the published version at https://doi.org/10.1007/s11082-021-03185-3. 


\title{
Diffraction of waves by a planar junction of impedance and perfect electromagnetic conductor half-planes
}

\author{
HUSNu DeNIZ BASDEMIR \\ Department of Electrical and Electronics Engineering, Konya Food and Agriculture University, Konya \\ 42080, TURKEY \\ denizbasdemir@gmail.com
}

\begin{abstract}
In this study, diffraction of waves by a planar junction between impedance and perfect electromagnetic conductor half-planes is investigated. The method of transition boundary is used for the solution of the problem. The diffracted wave expressions are expressed in terms of the Fresnel functions by using the uniform theory of diffraction method. Thus, finite magnitudes are obtained at the transition regions and the results are analyzed numerically for different set of parameters.
\end{abstract}

Keywords: Diffraction theory, Impedance surface, Perfect electromagnetic conductor, Scattering theory.

\section{Introduction}

In the literature, the diffraction of waves by a single half-plane is widely considered since it is a canonical problem. Besides, diffraction of waves by a junction which is formed by combination of two half-planes which have different boundary conditions is also an important problem. Because they have application in wave propagation over terrain, costal diffraction problem and analysis of wave interactions with vehicles that includes various material structures. Diffraction of waves by the junction of two impedance half-planes was first investigated for the special case of wedge diffraction (Malyuzhinets 1958). The impedance junction problem for the skew incidence case by using the Wiener-Hopf method was also investigated (Rojas 1988). The high frequency diffraction of plane waves by the junction of resistive and impedance half-planes was investigated (Uzgoren et al. 1989). The same problem was also investigated with the plane wave spectrum approach (Senior 1991). The scattering of waves by a planar junction of two half-planes with different combinations of surface boundary conditions can be found widely, but not limited to these studies, in the literature (Büyükaksoy et al. 2004; Gennarelli and Riccio 2020; Pelosi et al. 1998; Umul 2020; Umul 2017; Yalçın and Umul 2020).

In this study, we are dealing with the diffraction of plane waves by the planar junction between impedance and perfect electromagnetic conducting (PEMC) half-planes. As best as we know, such a kind of scenario has not been investigated yet. The existing studies in the literature does not include the combination of PEMC structures. The PEMC structure can be defined by the single admittance parameter $M$ where, the surface acts as a perfect electric conductor (PEC) when $M$ goes to positive or negative infinity, however the surface acts as a perfect magnetic conductor (PMC) when $M$ sets to zero. The analytical investigation of the problem under consideration is based on the recent method invented in a study (Umul 2019). His method, which is called method of transition boundaries (MTB) is based on the relations of scattered geometric optics fields and diffracted fields at the transition regions. In this study, the junction of half-planes with different boundary conditions is considered for the surfaces and the examination method is quite different than widely used in the literature. Therefore, this study considers a different case than the previous ones.

First, the problem will be introduced. The related geometrical optics (GO) fields will be determined, and initial fields will be derived considering the whole impedance plane. Then, 
scattered GO fields will be obtained by subtracting the initial fields from total GO fields where the total GO fields will be determined by using the geometry of the problem under consideration. Later, the diffracted fields will be derived by using a formulation between the scattered GO and diffracted fields at the transition boundaries. The ultimate diffracted fields will be expressed in terms of the Fresnel functions by using the uniform theory of diffraction (UTD) method. Finally, behavior of fields according to the angle of observation will be analyzed numerically for different set of parameters.

The time factor of $\exp (j \omega t)$ is suppressed throughout the paper where $\omega$ represents the angular frequency.

\section{Junction descriptions}

The diffraction geometry is given in Fig.1. The materials which cause the diffraction located at $y=0$ plane, and two different boundary conditions are considered. The cylindrical coordinates are represented as $(\rho, \phi, z)$ also angle of observation $\phi$ is assumed to be measured from the halfplane in the counter-clockwise direction. $P$ is the observation point and $\phi_{0}$ is the angle of incidence.

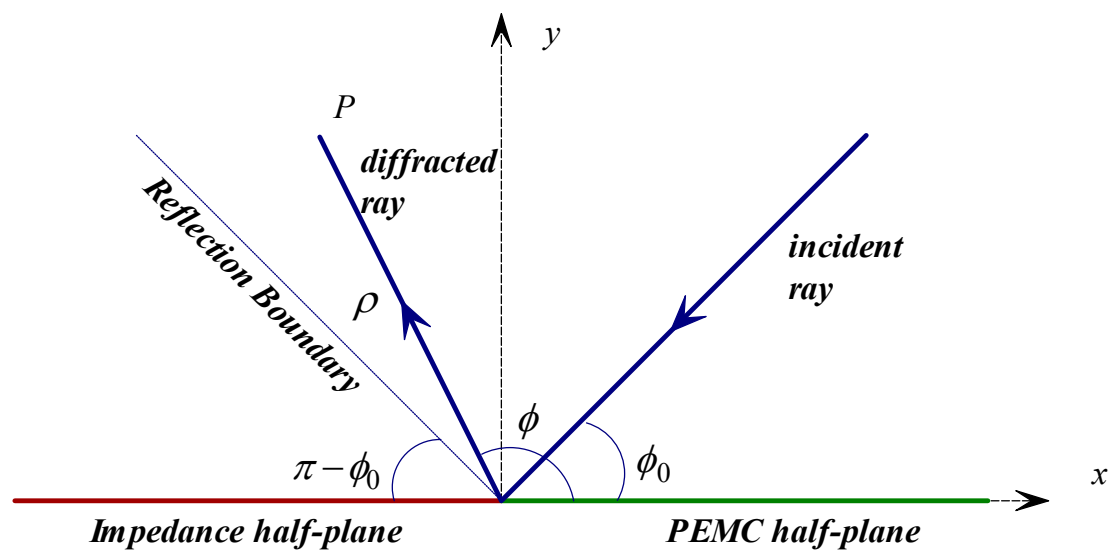

Fig. 1. The diffraction geometry

The impedance boundary condition imposed on $x<0$ of the plane $y=0$ refers to one of the half-planes. If the surface impedance is $Z$, the impedance boundary condition is

$$
\vec{n} \times \vec{n} \times\left.\vec{E}\right|_{\phi=\pi}=-Z \vec{n} \times\left.\vec{H}\right|_{\phi=\pi}
$$

where $n$ is the unit normal vector (Senior and Volakis 1995). The remaining part of the geometry is described with the boundary conditions of

$$
\begin{aligned}
& \vec{n} \times\left.(\vec{H}+M \vec{E})\right|_{\phi=0}=0, \\
& \left.\vec{n} \cdot\left(\varepsilon_{0} \vec{E}-\mu_{0} M \vec{H}\right)\right|_{\phi=0}=0
\end{aligned}
$$

where $E$ and $H$ terms define the total electric and magnetic field intensities. Also, $\varepsilon_{0}, \mu_{0}$ are the free space permittivity and permeability of the free space, respectively. Here, $M$ denotes the admittance of the half-plane (Basdemir 2019). The electric and magnetic field component of the incident plane-wave can be expressed as

$$
\left.\vec{E}_{i}=\vec{e}_{z} E_{0} e^{j k\left(x \cos \phi_{0}+y \sin \right.}{ }_{0}\right)
$$


and

$$
\vec{H}_{i}=\frac{E_{0}}{z_{0}}\left(-\vec{e}_{x} \sin \phi_{0}+\vec{e}_{y} \cos \phi_{0}\right) e^{j k\left(x \cos \phi_{0}+y \sin \phi_{0}\right)}(5)
$$

where $E_{0}$ is the complex amplitude.

\section{Determination of the $\mathbf{G O}$ fields}

In this part, the related $\mathrm{GO}$ fields with the reflection coefficients will be determined by considering the boundary conditions given above. The reflected electric and magnetic field intensities from PEMC and impedance surfaces are defined by

$$
\begin{aligned}
& \vec{E}_{r 1}=\left[-Z_{0} H_{r z}\left(\vec{e}_{x} \sin \phi_{0}+\vec{e}_{y} \cos \phi_{0}\right)+\vec{e}_{z} E_{r z}\right] e^{j k\left(x \cos \phi_{0}-y \sin \phi_{0}\right),} \\
& \left.\vec{H}_{r 1}=\left[\frac{E_{r z}}{z_{0}}\left(\vec{e}_{x} \sin \phi_{0}+\vec{e}_{y} \cos \phi_{0}\right)+\vec{e}_{z} H_{r z}\right] e^{j k\left(x \cos \phi_{0}-y \sin \right.} \quad 0\right),
\end{aligned}
$$

and

$$
\begin{aligned}
& \vec{E}_{r 2}=\vec{e}_{Z} \Gamma E_{0} e^{j k\left(x \cos \phi_{0}-y \sin \phi_{0}\right)}, \\
& \vec{H}_{r 2}=\frac{\Gamma E_{0}}{Z_{0}}\left(\vec{e}_{x} \sin \phi_{0}+\vec{e}_{y} \cos \phi_{0}\right) e^{j k\left(x \cos \phi_{0}-y \sin \phi_{0}\right)}
\end{aligned}
$$

where $Z_{0}$ is the intrinsic impedance of the free space and $\Gamma$ is the reflection coefficient of impedance surface (Basdemir 2021; Senior and Volakis 1995). The unknown components of reflected field intensities can be derived from the boundary conditions given in Eqs. (1), (2) and (3). Thus, the unknown components are given as

$$
\begin{aligned}
& E_{r z}=R_{z} E_{0}, \\
& R_{Z}=\frac{1-M^{2} Z_{0}^{2}}{1+M^{2} Z_{0}^{2}}
\end{aligned}
$$

and

$$
\begin{aligned}
& H_{r z}=R_{x y} E_{0}, \\
& R_{x y}=-\frac{2 M}{1+M^{2} Z_{0}^{2}}
\end{aligned}
$$

also

$$
\Gamma=\frac{\sin \phi_{0}-\sin \theta}{\sin 0_{0}+s i}
$$

where $\sin \theta$ represents the ratio of the impedances of free space and impedance half-plane, i.e. $Z_{0} / Z$. Then, it is possible to write the reflected GO fields considering the derived reflection coefficients. Thus, the total GO field expression for the electric field can be written by aid of the geometry of the problem as

$$
\vec{E}_{T G O}=\vec{E}_{i}+\vec{E}_{r 1} U\left(-\xi_{+}\right)+\vec{E}_{r 2} U\left(\xi_{+}\right)
$$

where $E_{r 1}$ and $E_{r 2}$ are the reflected electric field intensities from the surface of the PEMC half-plane and the impedance half-plane surface. $U(x)$ is the unit step function which represents the location of the fields in the related region in space. The argument of the unit step function is called as detour parameter and it is the ray path difference between GO and diffracted fields as

$$
\xi_{ \pm}=-\sqrt{2 k \rho} \cos \frac{\phi \pm \phi_{0}}{2} .
$$

The initial GO field can be written when the scattering object does not exist in the space. Thus, the initial GO field expression is obtained as

$$
\vec{E}_{\text {in }}=\vec{E}_{i}+\vec{E}_{r 2}
$$


when the junction which causes the diffraction vanishes and assuming the whole plane is consists of impedance surface. The scattered GO field can be found by subtraction of Eq. (17) from Eq. (15) as

$$
\vec{E}_{S G O}=\left(\vec{E}_{r 1}-\vec{E}_{r 2}\right) U\left(-\xi_{+}\right)
$$

by aid of the relation $U(-x)=U(x)-1$. The total magnetic field can be written as

$$
\vec{H}_{T G O}=\vec{H}_{i}+\vec{H}_{r 1} U\left(-\xi_{+}\right)+\vec{H}_{r 2} U\left(\xi_{+}\right)
$$

also, initial magnetic field intensity can be written as

$$
\vec{H}_{\text {in }}=\vec{H}_{i}+\vec{H}_{r 2}
$$

from the definition given above. Thus, scattered magnetic field intensity can be written as

$$
\vec{H}_{S G O}=\left(\vec{H}_{r 1}-\vec{H}_{r 2}\right) U\left(-\xi_{+}\right)
$$

by the subtraction of Eq. (20) from Eq. (19). In addition, keep in mind that the scattered GO fields have $x, y$ and $z$ subcomponents. Since the cross-polar components can be derived from the $z$-components of electric and magnetic field intensities, it is more suitable considering the $z$-components of scattered fields. Hence, $z$-components of scattered GO fields can be given as

and

$$
E_{S G O Z}=\left(R_{z}-\Gamma\right) E_{0} e^{j k(x \operatorname{co}} \quad 0^{\left.-y \sin \phi_{0}\right)} U\left(-\xi_{+}\right)
$$

$$
\left.H_{S G O z}=R_{x y} E_{0} e^{j k\left(x \cos \phi_{0}-y \sin \right.}{ }^{0}\right) U\left(-\xi_{+}\right)
$$

from Eqs. (18) and (21). There is not any contribution of the impedance surface to the $\mathrm{z}$ component of scattered GO field can be observed since the reflected magnetic fields from the impedance half-plane do not include the z-components.

\section{Determination of the diffracted fields}

In this section, we will derive diffracted fields by using the relation between scattered GO fields and diffracted fields at the transition regions. The general expression of the high frequency asymptotic expression of the diffracted field is given as

$$
E_{d}=E_{0} \frac{e^{-j \frac{\pi}{4}}}{\sqrt{2 \pi}} \frac{q\left(\phi, \phi_{0}\right)}{\cos \phi+\cos \phi_{0}} \frac{e^{-j k \rho}}{\sqrt{k \rho}}
$$

according to the geometrical theory of diffraction (GTD) and $q$ is the unknown function which can be defined by the relation of

$$
q\left(\pi-\phi_{0}, \phi_{0}\right)=-\left.\sin \phi_{0} A\left[E_{S G O}\right]\right|_{\phi=\pi-\phi_{0}}
$$

where $A[x]$ means that only consider the magnitude of $x$ by eliminating phase. Since the half-planes do not transmits the fields, there is only one transition boundary exists at $\pi$ - $\phi_{0}$ (Umul 2019). After the determination of unknown $q$ function, the diffracted field will be obtained. The unknown function $q$ is determined for $z$-polarized components of Eq. (22) as

$$
q\left(\pi-\phi_{0}, \phi_{0}\right)=-2 \frac{\sin \phi_{0}\left(\sin \theta-\sin \phi_{0} M^{2} Z_{0}^{2}\right)}{\left(\sin \phi_{0}+\sin \theta\right)\left(1+M^{2} Z_{0}^{2}\right)}
$$

where the expression can be rearranged as

$$
q\left(\pi-\phi_{0}, \phi_{0}\right)=-\left(R_{z}-1\right) \frac{\sin \phi_{0} \sin \gamma}{\sin \theta} \frac{K_{+}\left(\pi-\phi_{0}, \theta\right) K_{+}\left(\phi_{0}, \theta\right)}{K_{+}\left(\pi-\phi_{0}, \gamma\right) K_{+}\left(\phi_{0}, \gamma\right)}
$$

by multiplication and division of Eq. (26) with $\sin \phi_{0}, \sin \theta$ and $\sin \gamma$ and the relation of

$$
K_{+}(\tau, v) K_{+}(\pi-\tau, v)=\frac{\sin \tau \sin v}{\sin \tau+\sin v}
$$

where $K_{+}$is the split function which comes from the factorization process of Wiener-Hopf (Osipov and Tretyakov 2017). Also, the term $\sin \gamma$ is equal to $-\sin \theta / M^{2} Z_{0}^{2}$. The split function can be defined as 


$$
\begin{gathered}
K_{+}(\tau, v)= \\
\frac{4 \sqrt{\sin v} \sin ^{\tau} / 2}{\left[1+\sqrt{2} \cos \frac{\pi / 2^{-\tau+v}}{2}\right]\left[1+\sqrt{2} \cos \frac{3 \pi / 2^{-\tau-v}}{2}\right]}\left\{\frac{\psi_{\pi}(\pi / 2-\tau+v) \psi_{\pi}(3 \pi / 2-\tau-v)}{\left[\psi\left(\frac{\pi}{2}\right)\right]^{2}}\right\}
\end{gathered}
$$

where the function $\psi_{\pi}$, which is called as Maliuzhinetz function, can be defined by

$$
\psi_{\pi}(x)=\exp \left[-\frac{1}{8 \pi} \int_{0}^{x} \frac{\pi \sin t-2 \sqrt{2} \pi \sin ^{t} / 2+2 t}{\cos t} d t\right] .
$$

The function $q$ can be found by considering the principle of reciprocity as

$$
q\left(\phi, \phi_{0}\right)=-\left(R_{z}-1\right) \frac{2 \sin \frac{\phi}{2} \sin \frac{\phi_{0}}{2} \sin \gamma}{\sin \theta} \frac{K_{+}(\pi-\phi, \theta) K_{+}\left(\pi-\phi_{0}, \theta\right)}{K_{+}(\pi-\phi, \gamma) K_{+}\left(\pi-\phi_{0}, \gamma\right)}
$$

since the impedance half-plane located at the left side, the arguments of the split functions include $\pi$ - $\phi$ and $\pi$ - $\phi_{0}$ terms to satisfy the boundary conditions given above. After the determination of unknown $q$ function, the $z$-component of diffracted electric field can be written as

$$
E_{d z}=-\left(R_{z}-1\right) \frac{\sin \gamma}{\sin \theta} \frac{K_{+}(\pi-\phi, \theta) K_{+}\left(\pi-\phi_{0}, \theta\right)}{K_{+}(\pi-\phi, \gamma) K_{+}\left(\pi-\phi_{0}, \gamma\right)} E_{0} \frac{e^{-j \frac{\pi}{4}}}{\sqrt{2 \pi}} \frac{2 \sin \frac{\phi}{2} \sin \frac{\phi_{0}}{2}}{\cos \phi+\cos \phi_{0}} \frac{e^{-j k}}{\sqrt{k \rho}} .
$$

Since the high frequency asymptotic expressions of diffracted fields reach infinite magnitude values at the transition boundaries, diffracted fields must be expressed in terms of the Fresnel functions. Thus, finite magnitude values can be reads at the transition boundaries. The uniform field expressions are defined by the relation of

$$
\operatorname{sign}(x) F[|x|] \cong \frac{e^{-j \frac{\pi}{4}}}{2 \sqrt{\pi}} \frac{e^{-j x^{2}}}{x}
$$

where $\operatorname{sign}(x)$ is the signum function and equal to 1 when $x>0$ and -1 when $x<0$. Also, $F[|x|]$ is the Fresnel function which can be defined by the integral of

$$
F[x]=\frac{e^{j \frac{\pi}{4}}}{\sqrt{\pi}} \int_{x}^{\infty} e^{-j t^{2}} d t .
$$

The last terms of Eq. (32) are equal to the high frequency diffraction coefficient of soft halfplane (Basdemir 2018). Thus, it is possible to replace uniform version of last terms in Eq. (32), gives the uniform expression of

where

$$
E_{d z}^{u}=-\left(R_{z}-1\right) \frac{\sin \gamma}{\sin \theta} \frac{K_{+}(\pi-\phi, \theta) K_{+}\left(\pi-\phi_{0}, \theta\right)}{K_{+}(\pi-\phi, \gamma) K_{+}\left(\pi-\phi_{0}, \gamma\right)} E_{0} D_{s}
$$

$$
D_{s}=e^{j k \rho \cos \left(\phi-\phi_{0}\right)} \operatorname{sign}\left(\xi_{-}\right) F\left[\left|\xi_{-}\right|\right]-e^{j k \rho \cos \left(\phi+\phi_{0}\right)} \operatorname{sign}\left(\xi_{+}\right) F\left[\left|\xi_{+}\right|\right] .
$$

From now on, the diffracted magnetic field will be derived. It is clearly seen from Eq. (23) that the contribution to the scattered magnetic field only comes from the PEMC surface, since reflected magnetic field's intensity from the impedance half-plane does not includes the $z$ component of the magnetic field. However, such a kind of interpretation can only be acceptable in the situation when there is not any interaction of diffracted fields from the PEMC surface with the impedance half-plane or the interaction of currents flowing on the surfaces. It is known that each of the impedance and PEMC surfaces support both electric and magnetic currents. Therefore, it is reasonable to expect any interaction. If the effect of the impedance surface is neglected, the left side of the problem is accepted as an aperture. Such a kind of situation may cause incorrect diffracted fields to be derived for all junction problems. Under this circumstance, an alternative interpretation should be considered for determination of the diffracted magnetic field. The contribution of the impedance surface to the diffracted magnetic field can be revealed by considering the equivalent diffraction geometry which is given in Fig. 2. Here, the junction problem can be reduced to the impedance half-plane problem, by considering the incident magnetic field as $z$-component of reflected magnetic field from the PEMC surface, i.e. the $z$-component of Eq. (7) is considered as an incident magnetic field. Thus, 
the diffracted magnetic field in accordance with the Eq. (25) with the high frequency asymptotic expression of the diffracted field of an impedance half-plane (Umul 2019) can be obtained as

$$
H_{d z}=R_{x y}\left(1-\frac{2 \cos \frac{\alpha}{2} \cos \frac{\beta}{2}}{\sin \theta}\right) K_{+}(\beta, \theta) K_{+}(\alpha, \theta) E_{0} \frac{e^{-j \frac{\pi}{4}}}{\sqrt{2 \pi}} \frac{1}{\cos \beta+\cos \alpha} \frac{e^{-j k \rho}}{\sqrt{k \rho}} .
$$

and the uniform version of Eq. (37) can be obtained by multiplication and division of Eq. (37) with the $2 \sin (\phi / 2) \sin \left(\phi_{0} / 2\right)$. Thus, the same last terms of Eq. (32) are obtained. In this way, it is possible to reach uniform diffracted magnetic field by replacing the last terms of Eq. (37) with Eq. (36) as

$$
H_{d z}^{u}=R_{x y}\left(1-\frac{2 \cos \frac{\alpha}{2} \cos \frac{\beta}{2}}{\sin \theta}\right) \frac{K_{+}(\beta, \theta) K_{+}(\alpha, \theta)}{2 \sin \frac{\phi}{2} \sin \frac{\phi_{0}}{2}} E_{0} D_{s}
$$

where the angle values $\alpha, \beta$ are equal to $\pi-\phi_{0}$ and $\phi-\pi$ respectively according to the Fig. 2 . The scattered electric and magnetic fields are given by

and

$$
E_{S}^{u}=E_{S G O z}+E_{d z}^{u}
$$

$$
H_{S}^{u}=H_{S G O z}+H_{d z}^{u}
$$

by considering Eqs. (22), (23), (35) and (38).

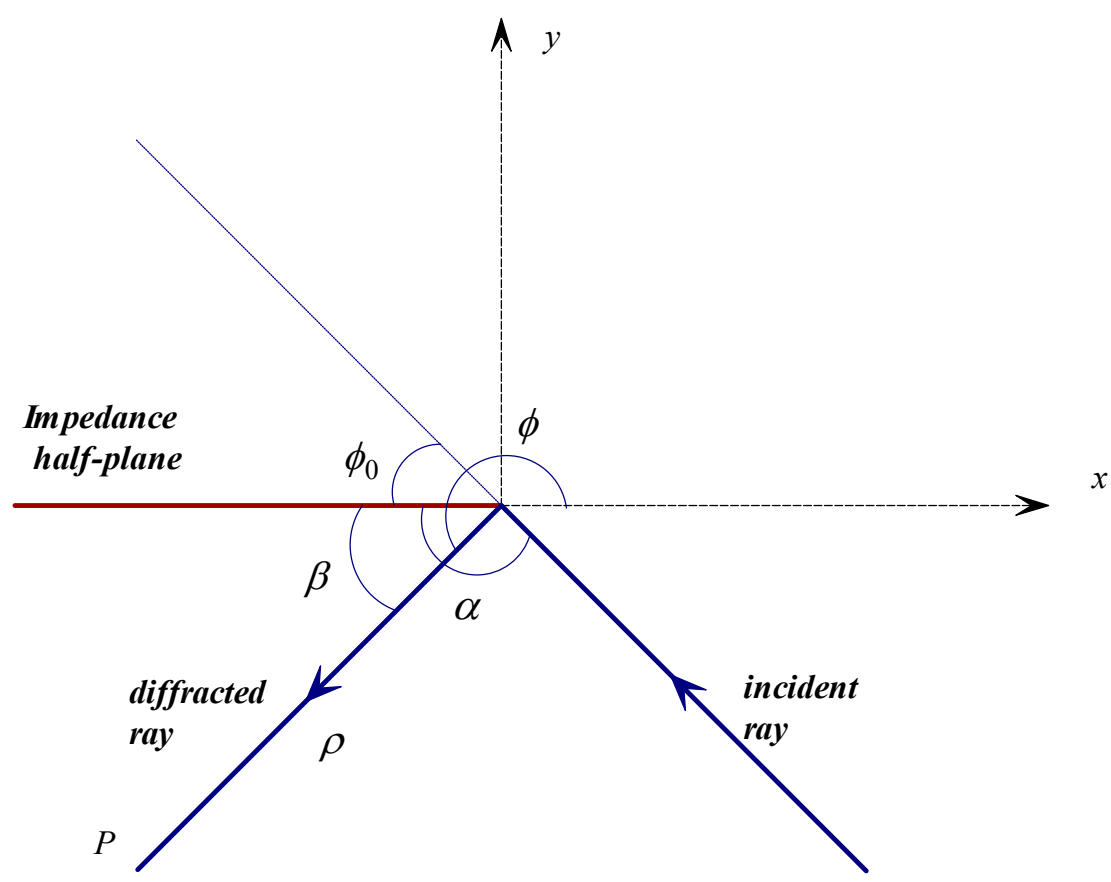

Fig. 2. The equivalent diffraction geometry

\section{Numerical results}

In this part, the magnitude variations of scattered, scattered GO and diffracted field values according to the angle of observation will be analyzed. The angle of incidence $\phi_{0}$ is taken as $60^{\circ}$ so the upper surface of both the half-planes is illuminated by the incident wave. The angle of observation varies between $0^{\circ}$ and $180^{\circ}$, which are indicating the upper surfaces of the planar 
half-planes respectively. Note that all the related parameters are normalized with respect to wavelength, $\lambda$. The wavenumber $k$ is taken as $2 \pi / \lambda$ where $\lambda$ is the wavelength and chosen as 0.1 for this problem. Also, the observation distance $\rho$ is taken as $6 \lambda$. The free space intrinsic impedance $Z_{0}$ is equal to $377 \Omega$ and the impedance of the half-plane $Z$ is taken as $10 \Omega$. The admittance $M$ is taken as 3 , unless otherwise stated.

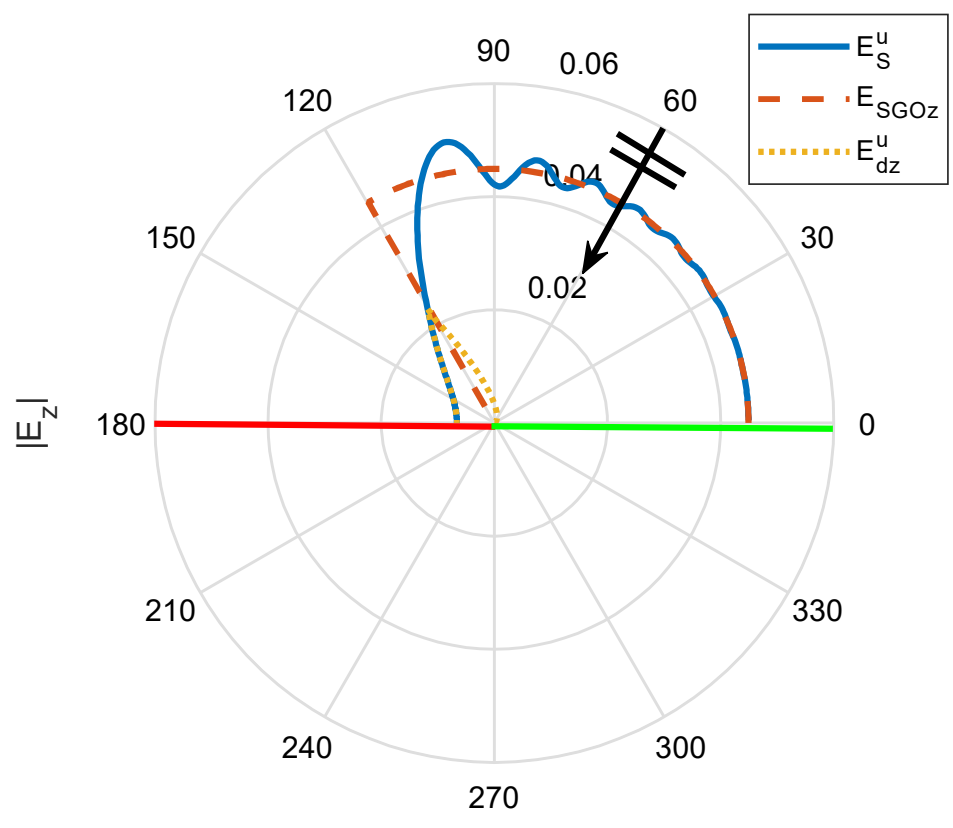

$\phi$

Fig. 3. The magnitude variations of $E_{z}$-components of scattered, scattered GO and diffracted fields with respect to the angle of observation $\phi$.

The $z$-component of the field variations are given in Fig. 3. It is seen that GO field has one discontinuity at the reflection $\left(120^{\circ}\right)$ boundary. It is acceptable since both half-planes do not transmits the fields. The diffracted field compensates this deficiency and reaches maximum magnitude values at the transition region. Thus, scattered field, which is consist of the interference of GO and diffracted field, is continuous at all observation angles. 


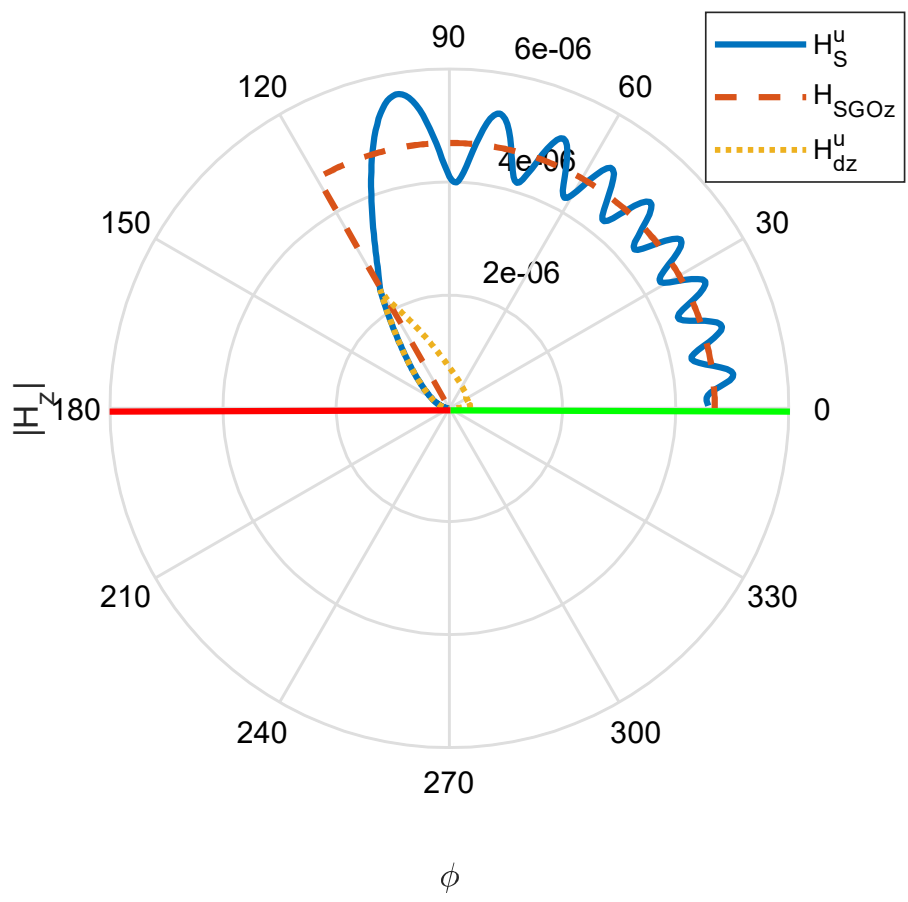

Fig. 4. The magnitude variations of $H_{z}$-components of scattered, scattered GO and diffracted fields with respect to the angle of observation $\phi$.

Figures 4 depicts the magnitude variations of $H_{z}$-components versus observation angle. The behavior of magnetic fields is similar with the electric fields. As expected, GO field is discontinuous at the reflection boundary and compensated by the diffracted field, thus obtaining a continuous scattered field on the considered path Although the units of electric and magnetic fields are different and do not allow comparison, the remarkable difference between their magnitudes from a mathematical point of view is due to the reflection coefficients. 


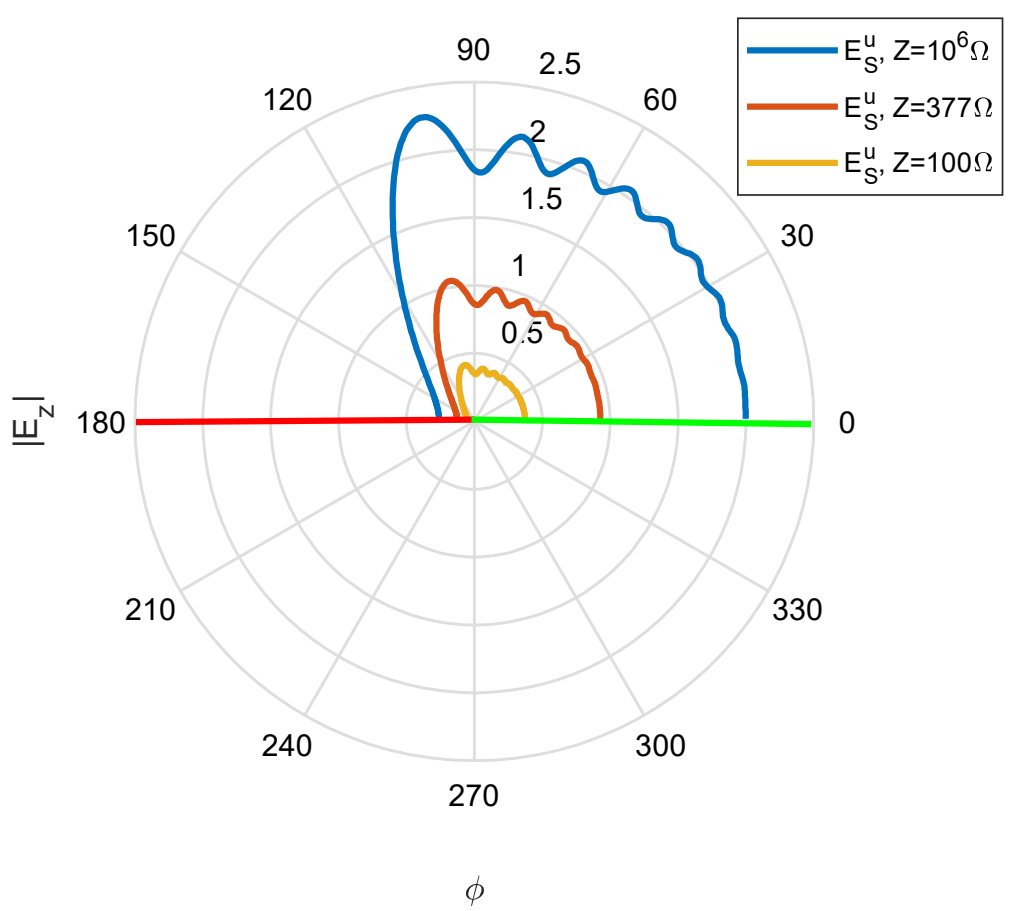

Fig. 5. Magnitude variations of the $E_{z}$-component of scattered field for different $Z$ values.

Figure 5 exhibits the magnitude variation of $E_{z}$-component of scattered field for different values of $Z$ versus the observation angle. The effect of $Z$ on the scattered field can be observed commonly all angle of observations. The term $Z$ is directly related with the reflection coefficient of the impedance half-plane given in Eq. (14). Thus, depending on the reflection coefficient, the reflected field from the half-plane is increasing when $Z$ increases. This means that when the $\mathrm{Z}$ increases the term $\sin \theta$ decreases, so the impedance surface goes to the perfectly conducting surface.

\section{Conclusions}

In this study, MTB method is used for investigating the plane-wave diffraction by a planar junction of two half-planes. The GO fields which have the discontinuity at the transition region were obtained by using the geometry of the problem. This deficiency was eliminated by the diffracted fields which were derived by using the relation between scattered GO and diffracted fields at the transition region. Thus, the total field was continuous in all directions of observation. Although the derived fields read infinite magnitude values at the reflection boundary, the infinities were eliminated by using the uniform theory of diffraction (UTD) method. To the best of our knowledge this study has not been tackled before. Finally, the related fields were examined numerically for different surface parameters. 


\section{Declarations}

Article received no funding.

There is no conflict of interests.

There is not any availability of data and material.

There is not any code availability.

\section{References}

Basdemir, H.D.: Modeling of an interface between isorefractive media by a resistive surface. Appl. Opt. 57, (2018). https://doi.org/10.1364/AO.57.001393

Basdemir, H.D.: Diffraction of waves by a perfect electromagnetic conductor half-plane between isorefractive media. Opt. Quantum Electron. 51, (2019). https://doi.org/10.1007/s11082-019-1947-0

Basdemir, H.D.: Magnetic line source diffraction by a conductive half-plane in an anisotropic plasma. Contrib. to Plasma Phys. 61, 1-10 (2021). https://doi.org/10.1002/ctpp.202000103 Büyükaksoy, A., Çinar, G., Serbest, A.H.: Scattering of plane waves by the junction of transmissive and soft-hard half-planes. Zeitschrift fur Angew. Math. und Phys. 55, 483-499 (2004). https://doi.org/10.1007/s00034-004-1065-z

G. D. Malyuzhinets: The excitation, reflection and radiation of surface waves in a wedge-like region with given face impedances. Dokl. Akad. Nauk SSSR. 121, 436-439 (1958)

Gennarelli, G., Riccio, G.: On the Accuracy of the Uniform Asymptotic Physical Optics

Solution for the Diffraction by a PEC-DNG Metamaterial Junction. IEEE Antennas Wirel.

Propag. Lett. 19, 581-585 (2020). https://doi.org/10.1109/LAWP.2020.2972308

Osipov, A. V., Tretyakov, S.A.: Modern Electromagnetic Scattering Theory with Applications. (2017)

Pelosi, G., Selleri, S., Manara, G.: Scattering from the junction of a perfectly conducting half-plane and a resistive sheet. Microw. Opt. Technol. Lett. 18, 85-86 (1998).

https://doi.org/10.1002/(SICI)1098-2760(19980605)18:2<85::AID-MOP1>3.0.CO;2-D

Rojas, R.G.: Wiener-Hopf Analysis of the EM Diffraction by an Impedance Discontinuity in a Planar Surface and by an Impedance Half-Plane. IEEE Trans. Antennas Propag. 36, 71-83 (1988). https://doi.org/10.1109/8.1076

Senior, T.B.A.: Skew incidence on a material junction. Radio Sci. 26, 305-311 (1991). https://doi.org/10.1029/90RS02684

Senior, T.B.A., Volakis, J.L.: Approximate Boundary Conditions in Electromagnetics. Approx. Bound. Cond. Electromagn. (1995). https://doi.org/10.1049/pbew041e Umul, Y.Z.: Wave diffraction by the junction between resistive and soft-hard half-screens. Optik (Stuttg). 130, 750-756 (2017). https://doi.org/10.1016/j.ijleo.2016.10.133 Umul, Y.Z.: The method of transition boundary for the solution of diffraction problems. Opt. Quantum Electron. 51, 1-15 (2019). https://doi.org/10.1007/s11082-019-1896-7

Umul, Y.Z.: Diffraction of electromagnetic waves by a resistive half-plane in magneto-ionic plasma. Contrib. to Plasma Phys. 60, 1-17 (2020). https://doi.org/10.1002/ctpp.202000009 Uzgoren, G., Buyukaksoy, A., Serbest, A.H.: Diffraction coefficient related to a discontinuity formed by impedance and resistive halfplanes. IEE Proc. H Microwaves, Antennas Propag. 136, 19-23 (1989). https://doi.org/10.1049/ip-h-2.1989.0003

Yalçın, U., Umul, Y.Z.: Scattering of waves by a planar junction between conductive and impedance half-planes. Optik (Stuttg). 203, (2020).

https://doi.org/10.1016/j.ijleo.2019.163943 
Figures

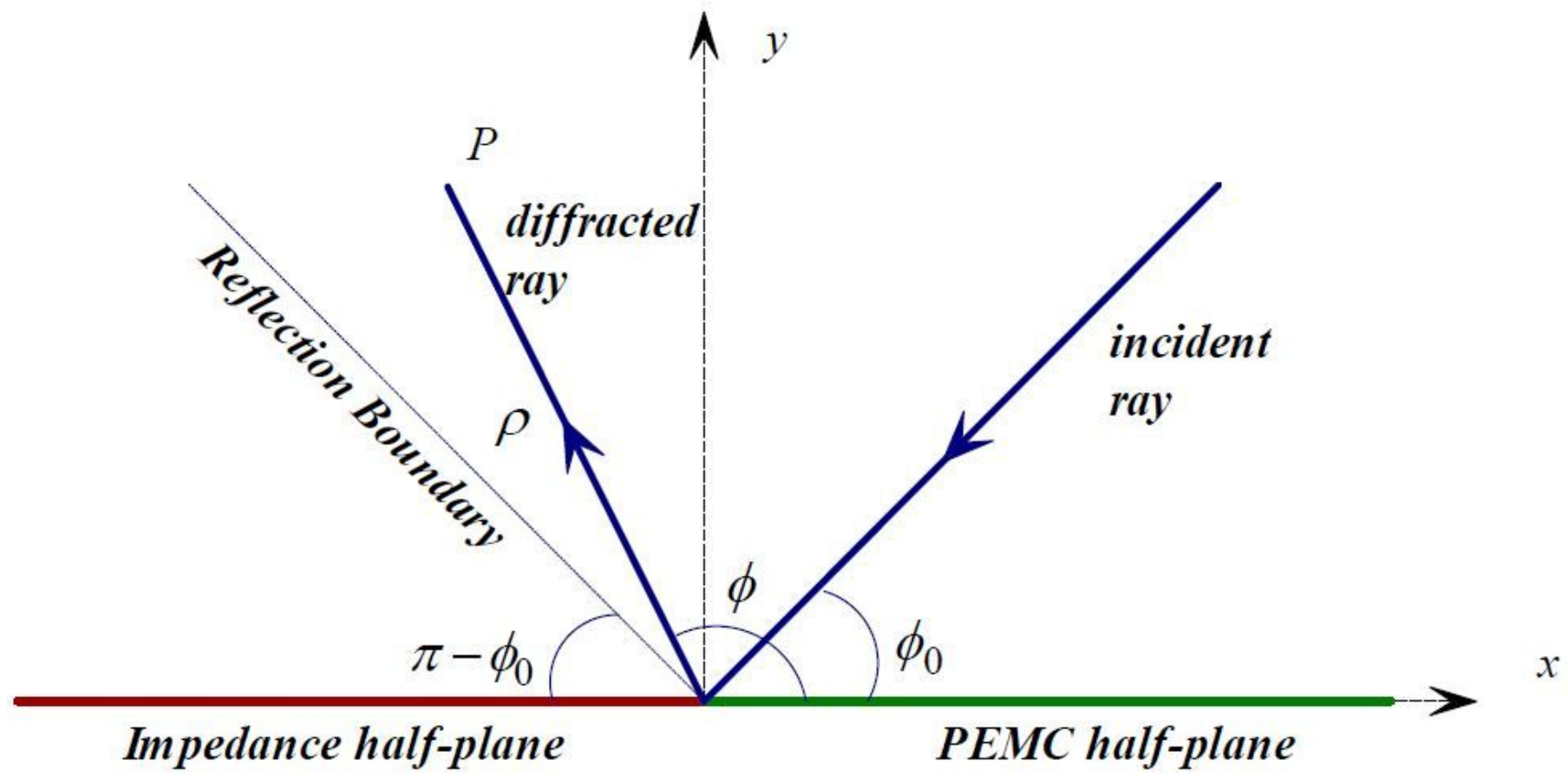

Figure 1

The diffraction geometry 


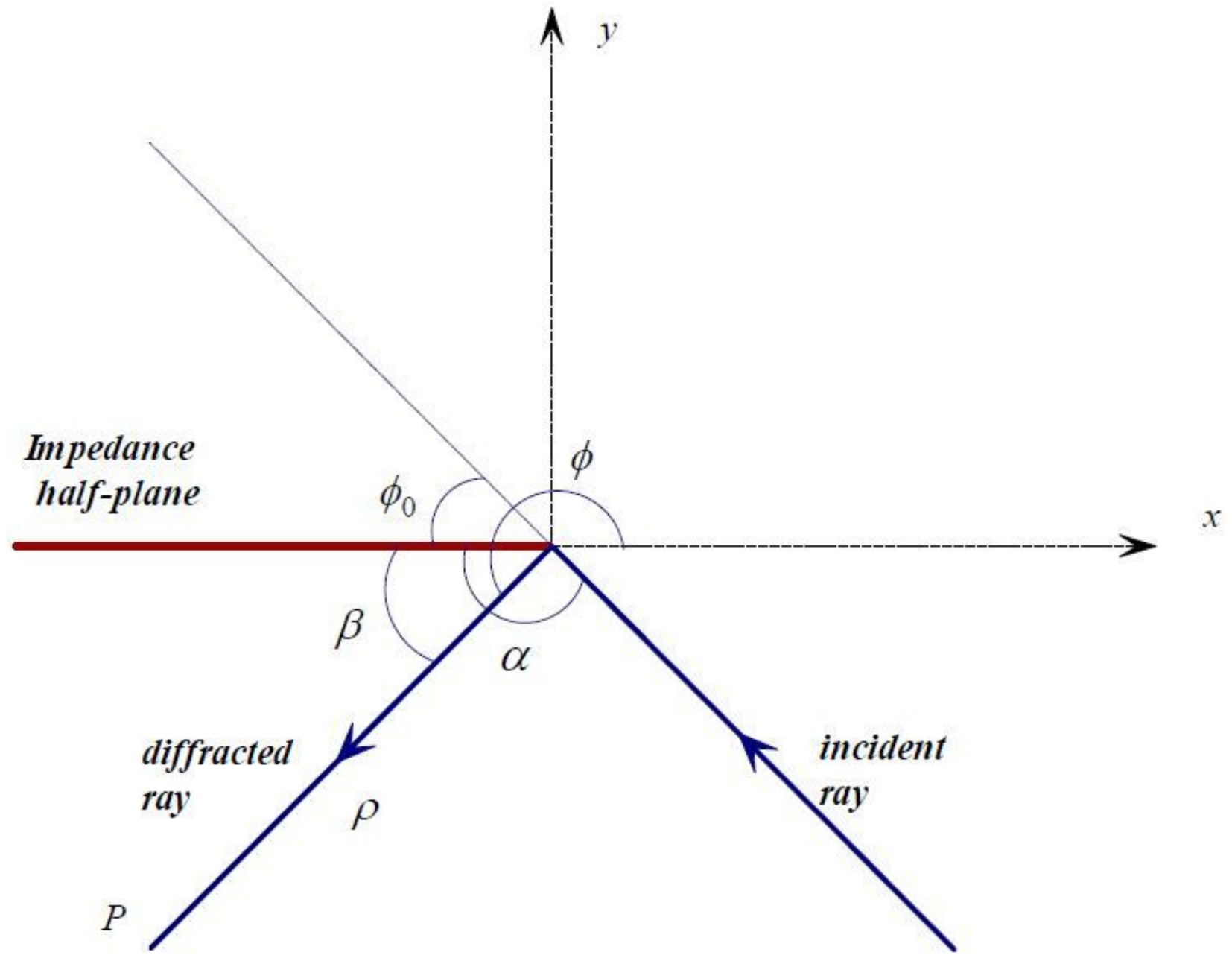

Figure 2

The equivalent diffraction geometry 


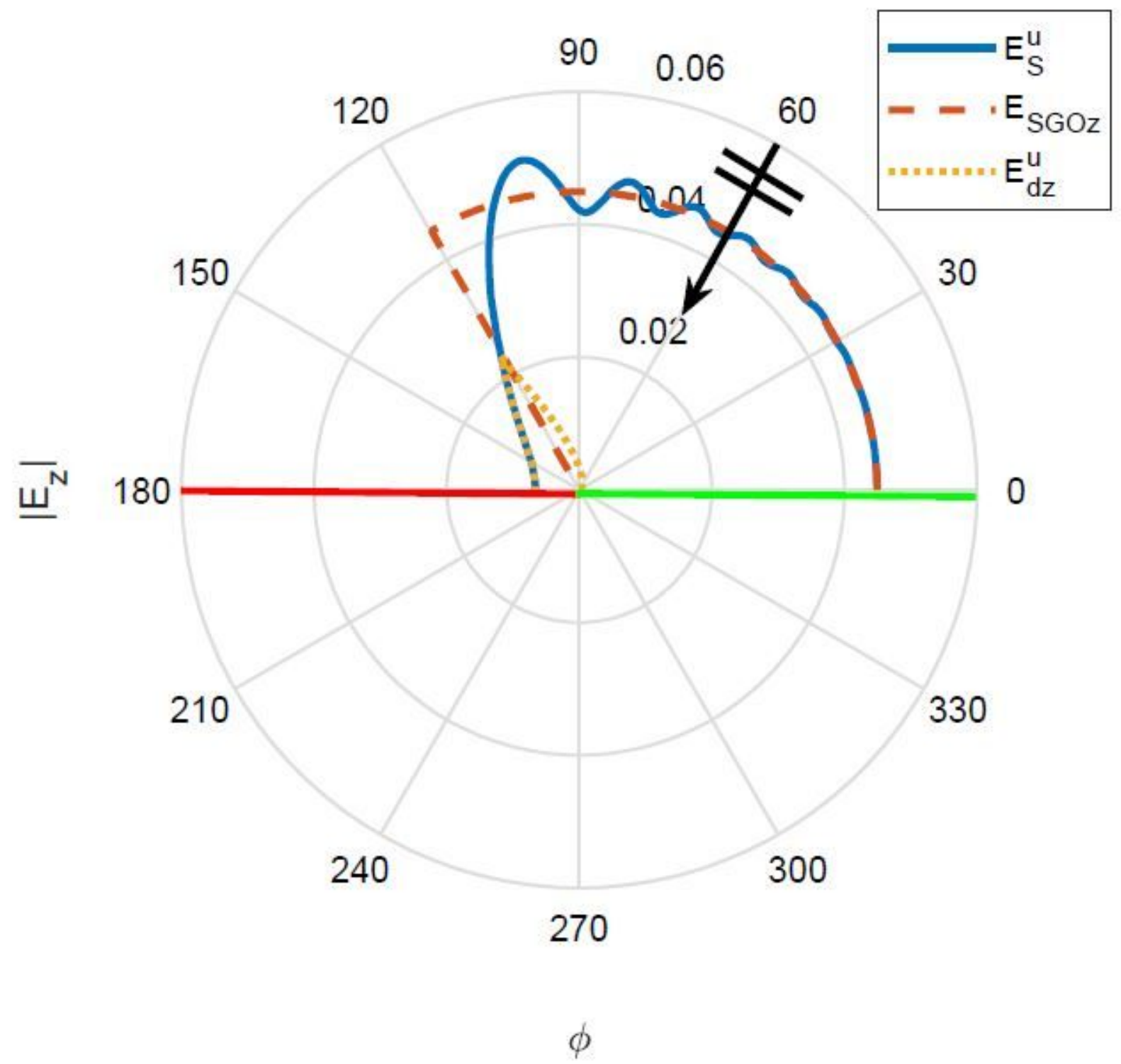

Figure 3

The magnitude variations of Ez-components of scattered, scattered GO and diffracted fields with respect to the angle of observation $\otimes$. 


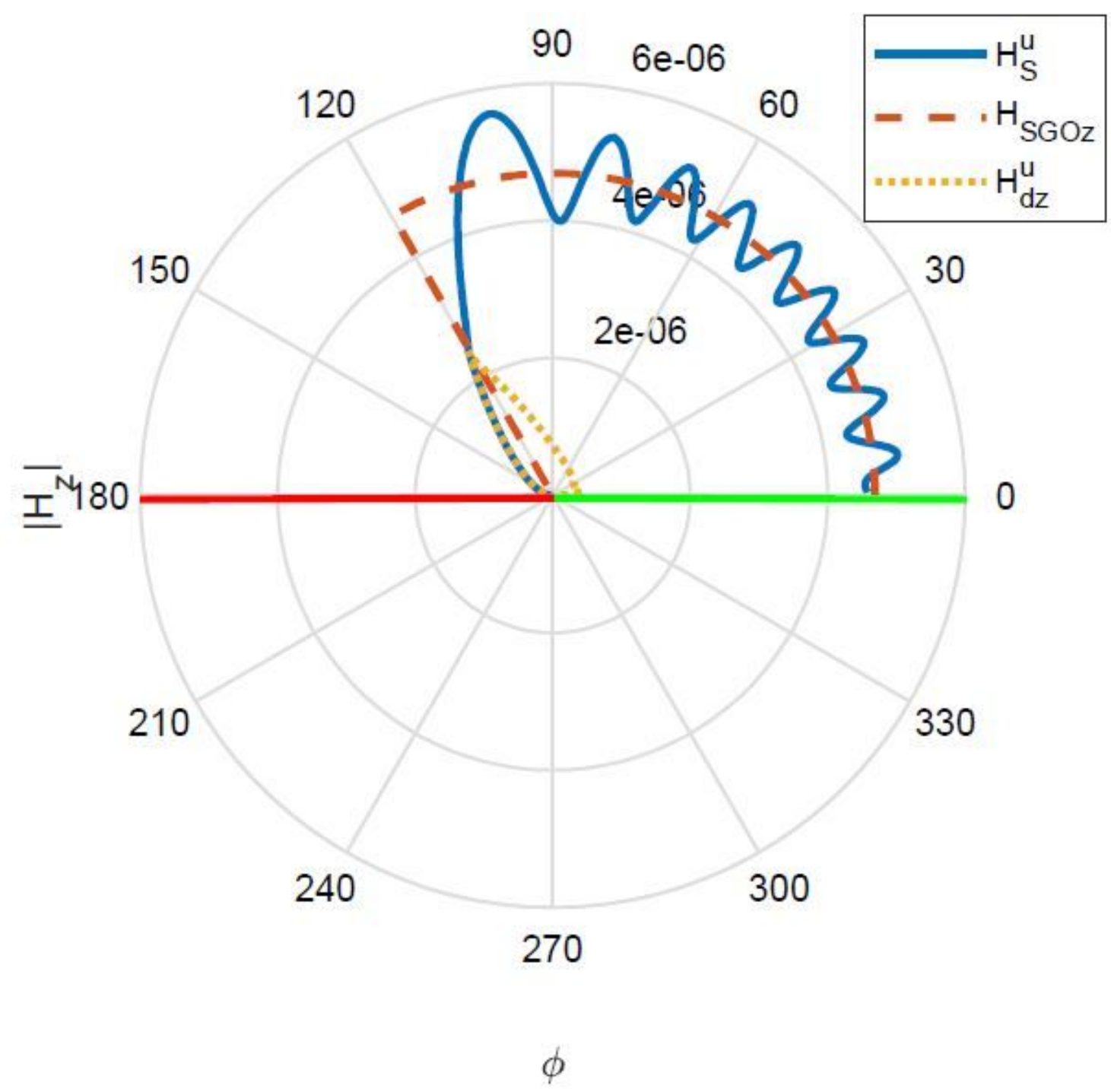

Figure 4

The magnitude variations of Hz-components of scattered, scattered GO and diffracted fields with respect

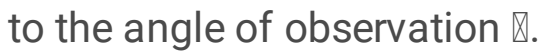




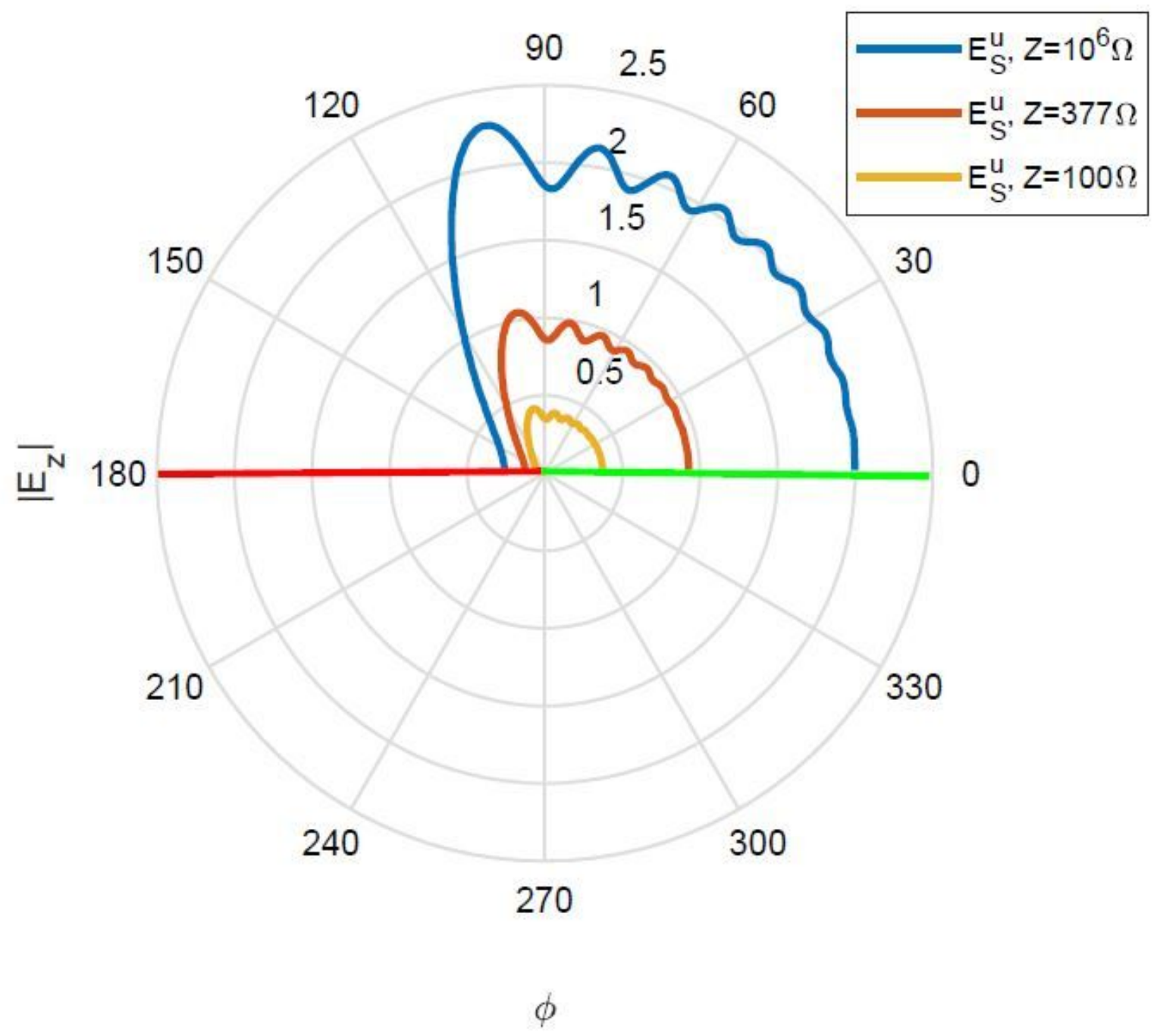

Figure 5

Magnitude variations of the Ez-component of scattered field for different $Z$ values. 